\title{
Zum Glossar von Belmont.
}

(Zu Zeitschriit XXXIII, 385 ffg.; XXXIV, 162 ffg.)

Was ich während eines neuen vierwöchentlichen Aufenthaltes (im August 1910) in Belmont an Nachträgen und Besserungen gesammelt habe, wird hier mitgeteilt: fast alles verdanke ich wieder Herrn Charles Morel und Frau Rosine Scheppler. Die beiden früher in dieser Zeitschrift erschienenen Beiträge bezeichne ich mit XXXIII und XXXIV.

ax la, nicht axta (XXXIII, 387).

badermōn f. fenouil, Fenchel.

begdajle, nicht bẹdāi (XXXIII, ${ }^{8} 89$ ). bĕn war früher auch bekannt als

Bezeichnung des Korbwagens

für Holzkohlen.

bene $e^{i}$ und bene (XXXIII, 389): on

$y$ met des cuveaux, du bois, rarement $\mathrm{du}$ foin.

beriā, f. berizer garçon, fille qui crie beaucoup.

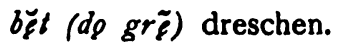

b̆̌ta (a/d) m. auch porte de la rä; s. auch $15 \bar{a} s$ s.

bikkle bezeichnet le premier binage

des pommes de terre, hifle

le second: beides nur von den

Kartoffeln; sfrkif dagegen nur vom Roggen.

brž̆ m. fiente d'enfants, de chats, chiens, pti b., gra $b$.

brtāi, en botai $d$ kufse sa partie

de chair avec du sang caille;

s. küşš̆ v. lï. buāija, f. buạajōr celui, celle qui bâille (zu buạiii).

buạlăt richtiger als buălat (XXXIII, 390).

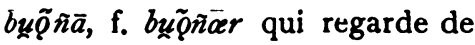
travers, sournois; bũōñär d'une vache qui regarde de travers, d'un air menaçant.

buõni, 3. buọñei $(a / \delta)$ regarder de travers.

brajāe m. auf dem Erdboden aufstehender Verschlag aus Brettern, in dem der Mörtel (mäll) bereitet wird (aus Kalk, Sand u. Zement); in der kfs de $t s \bar{a} \bar{s} \sin$, einer Mulde aus Holz, wird die malt nach der Arbeitsstelle gebracht; s. noch fregatat.

bra, il ẹ vnü fer $i$ bra sagt man von dem Hausherrn, der auf den Acker kommt, um mit Hand anzulegen und seine Leute zur Arbeit anzutreiben 
(pu pusi; in diesem Sinne pusi, nicht busi).

brause se dit du pus, de l'eau qui coule abondamment; sa brau, saz è brauce.

brāų f. endroit mou que l'eau traverse.

dãsi tanzen.

dãsu, däsrăs Tänzer, Tänzerin. dedo s. färę.

dexi $\bar{a}$, f. dexīer celui, celle qui gâte une chose; să en dexiāer d'une vache qui gâte le foin:

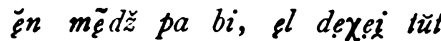
(alles).

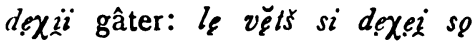
fúa $(\hat{a} \mid \delta)$.

dexteible, 1. dexteibgl se dit d'une mauvaise ménagère: gaspiller, donner à tort et à travers, $\mathrm{p}$. ex. des pommes de terre, sans que le mari le sache.

dextęblēer femme mauvaise ménagère, qui gaspille.

der lq $H_{\breve{q} t}$ derrière la Hutte

(Annexe von Belmont). deri, l ăx $d_{f} r i$ la porte de derrière. dẹsa'hnāi (= désaisonnée): el a trọ d. von einer Kuh, die zu spät kalbt (dies heifst auch fl s edadž, el a edadžei); die richtige Zeit zum Kalben ist Dezember bis Mai. Die Tiere, welche im Sommer kalben, kann man nicht auf die Weide schicken (li lātši $o$ peçküi) und verkauft sie deshalb lieber.

detšeias, le lün detšejas ist im Abnehmen begriffen, nicht $l_{g}$ lin e detseeia (XXXIII, 393), oder el detsa.

detša s. detseiads.

deva, l ă $\chi$ deva porte d'entrée. $d \bar{o} \underline{i}$ doppelt, $s$ a dọ $\bar{i}$; lo dọ $\dot{q}$ das

Doppelte; aber dreifach tra fu axta.

dọịi verdoppeln, vgl. erdoịiXXXIII, 396.

drăhā $l$ alāi in dem Hausflur, und so auch besser $d r a ̆ h \bar{a}$ in einem Wort XXXIV, 164. drăs le d'une échelle qu'on met debout (dresse la).

$d r \bar{o} m \mathrm{~m}$. gros abcès qui suppure (mot qui disparaît).

džalad f. dévidoir; so hörte ich zuletzt, nicht džalän (XXXIII, 394).

$d \check{z} \bar{q} r$ m. Schwiegersohn; so hörte ich zuletzt, nicht $d z ̌ \tilde{g} r$ (XXXIII, 394).

džgrme keimen, nicht dž̨̧rmi (XXXIII, 394).

dž $\mathrm{m}$. die gleichzeitig von mehreren Arbeitern in Angriff genommene Reihe auf dem Acker, die mehr oder weniger breit sein kann je nach der Zahl der Arbeiter und der Grölse des Feldes: lo $d \check{z} \bar{i} a$ $h \bar{a}$ die Reihe ist beendet, erledigt, weil man in dem Berglande von unten nach oben aufsteigend arbeitet; - auch der von dem Dachdecker auf einmal in Angriff genommene Teil des Strohdaches, das aus mehreren parallelen Lagen besteht. 
$d z ̌ \underline{o} t$ f. bezeichnet aufser dem Kohl, besonders dem chou pour faire la choucroute, auch les fanes des navets, pommes de terre, terleps; brás sind ausschliefslich die tanes sèches des pommes de terre.

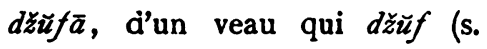

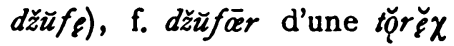
qui se jette dans les jupes d'une femme, comme pour chercher à boire, $s \breve{a} \breve{g} n$ ŭğt $d \check{z}$. $d \check{z u u f f}$ und $d \check{z} \breve{u} f$, d'un veau qui donne contre le pis de la vache pour l'attraper: $s \breve{a} i$ ŭğt $d \breve{z} \breve{u} f \bar{a}, i$ n fe ri ke $d \check{z} \breve{u} f$; auch

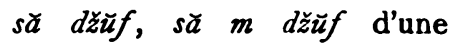
douleur sourde, d'un élancement quand le sang bat dans un abcès.

zur: man sagt bo žur, guten Tag', nicht b.džg; vgl. suar.

$\& b \bar{a} i$ f. envie, Gelüste, $d \check{z} \& \& b \bar{a} i$ $d$ als.

edādži hat als Partic. fem. \&dādžę, fl a \&. sie hat sich verspätet; von Früchten die spät reifen: i edadzi kmă, en edādžfi pür (Birne): frühreif ist to meĭ̄, mejar; s. noch desa'hnāi ede; $f \&$ mek $\& d e$ continue toujours, ve $t \tilde{\&}$ \&de continue à marcher; ein dem frz. continuer entsprechendes Wort besitzt die Mundart nicht.

\&dō (i s \&.) schläft ein. efuz\&, f. efuzāi von Salat, Rüben, Mohrrüben, die ins Kraut schiefsen und nicht mehr zum Essen taugen.

chodle déchiré, brisé ist XXXIII, 395 zu streichen.

expāl Schulter. expuvate épouvanter.

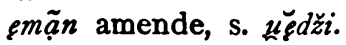

épettsi empêcher.

çvęi envie (de manger), s. $\notin b \bar{a} i$.

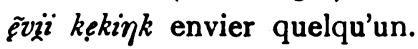

eusdži parier, $i$ po $d v i$ un pot de vin.

encedzāer f. pari.

erbüsi: I. erbüsi lo ts̆ă faire reculer le char, 2. $\check{z} m \&$ rbĭsi lo br\&, l expāl je me suis démis le bras, 3. $\check{z} l$ \& rbüsi soo siz spęm ich habe sie mit ihren Himbeeren abgewiesen, 4. să pü vu rbüsi, să me rbüs cela me dégoûte, ekelt mich an. eri, li măt fri ìk d lat les éloigner l'un de l'autre; v\& $t \tilde{\varepsilon} \& r i$ éloigne-toi.

erix s. lis.

erleve (1. erlev) di piăer ramasser des pierres; se dit des choses qu'on conserve, met de côté, pour qu'elles ne gênent pas: on erlegv lo $\chi$ o, le chaume du toit' dans les rẹg.

erole, fā grolg lo bo tsü lo gerne: das Holz so aufsetzen, dals es wohl geordnet ist, keinen unordentlichen Haufen bildet.

eru'hi, I. gru'h aiguiser.

ersane, I. ersan, ressembler, mit Acc. und Dativ: $i$ vï ersane se pẹr und \& se pẹr. 
ertšğdzi, lo to v\& \&. le temps va changer.

erveni, $t$ grve ma neben te ve moe es geht dir besser.

esăme assommer.

ĕsmuiii (s $\left.\breve{c}_{e}\right)$ ), 3. is esmuı, avoir envie de dormir, nicht s'endormir (XXXIII, 397); s. edọ. et $\tilde{a}$ Weiher ist feminin wie auch in $\mathrm{La}$ Baroche.

\&val $d_{\xi}$ dọ le fās gữōdz oder do lq gūogdz de demuadž la gorge de dimanche, sich verschlucken (avaler de travers).

fadi m. s. lí.

fārai f. choc, poussée, Substantivum zu

făr donner, pousser contre quelqu'un: il e vnü färe kotr mi; $i$ s a färe i $k$ dedo $l a \bar{t}$.

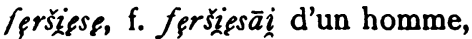
d'une femme qui se comportent, comme s'ils étaient toqués.

$f_{e} r t a ̄ l b \ell$, fertălbāi (elsäss. verdarben), nur von Tieren: d'un bœuf, d'une vache mal nourris. fi $d$ ts $u \bar{a}$ Pferdekot.

filfilat f. petit bouton percé de trous: on y fait passer une ficelle qu'on tire pour jouer. filrăs (\&n) femme qui file. figs auch von Beeren, die einschrumpfen (ratatiné). fots $\bar{i}$, il $\breve{a} h \bar{a}$ frts $i$ von einem grofsen Manne (haut sur ses

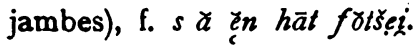
$f \breve{u}, m_{\xi} f \breve{u}$, par $m \xi f u ̈$ (foi) sagen die Männer, die Frauen $m_{\xi}$ frik; dagegen l\& fü $o b \sigma$ dice.

fuarg beschlagen, $f . i$ bü ferrer un bœuf; schlagen: il $i \in t i i$ fuarg; fuar lo düx (frappe ferme).

frạ, kalt; $s$ a mek $i$ fră mü n'est qu'un mur fait de pierres superposées, sans ciment.

frạ̄itrọ̆ m. Mistkäfer.

frālā (gro f.), f. fralēer, Substantiv zu fräle.

frālāi f. tas de bois en désordre, non rangé; le frālāi $n$ a pa $b \bar{i}$; il a m\&k \& frāla $\bar{a}$; nicht $\mathbf{z u}$ verwechseln mit frălq s'ébouler XXXIII, 400.

frālat f. flüssiger Kot von Kindern,

Hühnern, en grọs $f$.

frălg péter und foirer.

frătíu, f. frăliūuz d'une étoffe qui s'effiloche.

freiat f. planchette munie sur le revers d'une poignée, sur laquelle le maçon met le mortier qu'il applique avec la $\chi$ teruel; s. braje.

ğr ry f. Verschläge im Keller zu ebener Erde, zum Sortieren der verschiedenen Arten Kartoffeln, Rüben, usw.

gülăt von einer Ziege, während gizlü vom Zicklein üblich ist: vi, me gìlăt; s a not gilăt, nột gilu.

gūōdž s. gvale.

grāz Gras mit der Hand ausrupfen. 


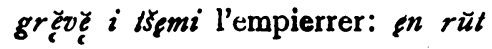
grevāiz; vgl. tsădži.

gržiz égratigner (avec une épine): $z m$ \& grifi; lo tṣ̌̆ $m$ ẹ grifi; Partic. fem. (dž \& lï) grifei.

haus bellen.

hargot (lp ts $\left.s_{a}\right)$ d'une voiture qui est cahotée.

$h \bar{e} b l$ s. $l i$.

hধ̨nă m. Teil des Webstuhles

(métier à tisser le ruban): parallel laufende mit Fäden überspannte Holzstäbchen, $b a$ get do hernă = morceaux de bois avec la lice.

$h e ̨ t s i$ Infin. hacher.

hẹttsi m. latte (manchmal sind es zwei) clouée entre deux poutres pour y remiser (in horizontaler Lage) des haches, des pioches, usw.

hokd renvoi, Aufstolsen.

holø cri pour arrêter les bœufs. huble $\mathrm{m}$. tertre, mamelon, monticule sur un haut plateau, couvert de myrtiles, d'airelles, etc.; vgl. kraxte.

hurg (fl h.), (imparfait) d'une poule malade qui se ramasse sur elle-même sous la pluie.

$\chi a k \bar{e} r$ f. brûlure légère; vgl. $\zeta ð k$.

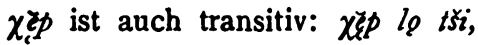

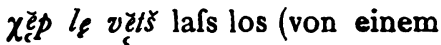
bösen Tier, das man angefarst hat): dagegen lātš lo tši den Hund von der Kette lassen; lāts $l_{\ell} v \underline{t c t} t s$ auf die Weide hinauslassen; vgl. rexep. $\chi \& r g \breve{\psi} t$ ist auch der Name einer Waldung; der Bach (vgl. XXXIV, 176 v. Chirgoutte) heifst noch rï̈ $d$ sẹ̣ nach einer Sägemühle.

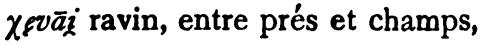
hors de Belmont près du Trou, où se déverse l'égout du village (wohl excavata).

$\chi \notin v a ̆ t$ f. écheveau; $\chi$. de tręm écheveau pour la fabrication du ruban.

$\chi^{m a ̋ k}$, sa $\chi$. bug riecht gut.

$\chi^{n u ̈} d r, \chi^{n o ̣} d r \mathrm{~m}$. morve du nez

(vgl. $\chi$ nodru XXXIII, 405).

$\chi \gamma d \xi$ d'un homme qui a l'oreille dure et qui en même temps est sournois, d'un esprit peu éveillé.

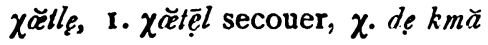
ligar, durcheinander schütteln, um sie $z u$ waschen, von Unreinigkeiten zu säubern.

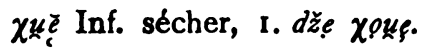

xpïri (se $\chi$.) von Hühnern, die die Federn verlieren, mausern; fl ve s xpüri, 3. xpür, 6. se

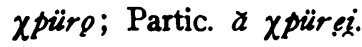
$\chi^{\text {tadle }}$ stre debcut, 1. dze $\chi^{\text {taldel, }}$

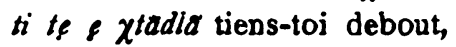
fā $t$ teni \& $\chi_{\text {tadla. }}$

$\chi^{t z k} k_{g}$ saigner un porc, lui enfoncer le couteau jusqu'au cœur, abstechen; vgl. $\chi^{\hbar z k a \bar{a} j}$ XXXIII, 406.

$\chi^{t \bar{\rho} d,} 3 \cdot \chi^{t \bar{o}}$, Partic. $\chi^{t \bar{o} d \ddot{~}}$ tordre. $\chi_{c}^{t u ̆ p}$ f. étoupe; s. $l_{i}$.

$i$ pleonastisch in $i l$ i $\breve{e} t u ̈ z \breve{u} k \breve{e}$, il i. e tü fuare. 
kataplä de linüz = cataplasme. $k$ kruāi f. war immer eine freiwillige Leistung für die $\mathrm{Ge}$ meinde, zu der sich auch die Frauen erboten; die Pfichtleistungen, die mit Geld ablösbar sind, heifsen prestations. $k$ ęrtg nicht schlagen im allgemeinen, sondern corriger, fustiger un enfant.

kess de tsāsin s. braija.

kĭe auch von der Frucht, die dünn steht, clairsemé.

$k l u ̈ k g, 6 . k l u ̈ k g$, d'une poule qui veut couver.

$k l u ̈ k \bar{a} r$ neben $k l u ̈ k$ poule qui veut couver.

knole Mehlklörschen oder Klümp-

chen, die mit dem Sieb (ridž) herausgesiebt werden.

$k \grave{o ̣ d r}$ f. (elsäfs. Kóter) crachat qu'on expectore lorsqu'on a la toux.

kŏla, dż a to kola do mi solg (buchstäblich: je suis tout coulant dans mes souliers) bin ganz durchnärst, auch $d z$ a pis $d$ au do mi sole.

kole m. Milchsieb; pect do kole linge dont on l'entoure, um Unreinigkeiten aus der Milch auszuscheiden, die das kole durchlärst.

kgtre $(k \breve{u} t r)$ f. grande bouteille entourée d'osier, de $\chi^{i n} \delta$, d'une contenance de 20 à 30 litres.

$k \boldsymbol{c}^{\prime} h \tilde{a} \mathrm{~m}$. (Lokalfranzösisch fer chaud) Sodbrennen, brûlure qui monte de l'estomac lors- qu'on ne digère pas bien ou qu'on se baisse en piochant. $k u ̈ t_{s}$ f. bezeichnet einmal alle efsbaren Pilze, soweit sie nicht besondere Namen haben wie morix, rieli, menăt; dann eine besondere efsbare Art, die coucoumelle (quand ceux-ci sont petits et pas ouverts, on les appelle prtð); die Giftpilze nennt man muar küts.s.

kugh he $\mathrm{m}$. estomac de veau ou de cabri dont on se sert pour faire cailler le lait.

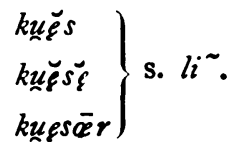

kră m. la crête, le point le plus élevé, culminant d'une côte, et tsü lo kră: doch wird nicht jede Anhöhe eines beliebigen Berges so genannt, sondern das Wort wird gleichsam als Eigenname nur 4-5 Höhen um Belmont beigelegt: zwei auf dem Hochfeld ( $k r \check{a} d \rho$ tsã und kră di brẹbli), dann lo kra di tsa di mo, lo kra der If $H \breve{q} t$ und ein kră bei den tsa Salomó.

kraqa m. douleur que les jeunes gens ressentent à l'aine à la suite de la croissance; ,envie', petit filet qui se détache entre l'ongle et la peau.

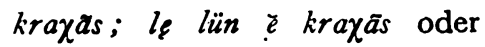
gl $k r a ̆$ ist XXXIII, 4 Io s. v. sră $\chi$ einzusetzen statt $l_{f}$ lin $\bar{c}$ kraxa. 
krăxte m. ist Deminutiv zu kra, also kleine Anhöhe; aufserdem bezeichnet es dasselbe was huble tertre, mamelon, tumulus, die leichten mit Heidekraut, mit Heidel- und Preifselbeeren bestandenen Bodenanschwellungen auf einer Hochebene oder einer Anhöhe.

lâtign $\mathrm{f}$. forme que prennent les morceaux de bois symétriquement rangés avec des intervalles, de manière à laisser passer l'air pour qu'ils sèchent mieux.

launi, louăt XXXIII, 4 I I sind nur wenigen bekannt.

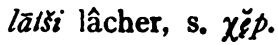

lf Artikel und Pronomen feminin singular: If vănla la voilà.

lev, lo stö lev die Sonne geht auf.

li Artikel und Pronomen maskulin und feminin plural, vor Vokal lis, li vănsi, li vănla = les voici, les voilà.

$l^{\sim} \mathrm{m}$. Flachs wurde früher in Belmont gebaut und verarbeitet, heutzutage jedoch nicht mebr. Die nur noch von wenigen gekannten, auf den Flachsbau und die Flachsbearbeitung bezüglichen Ausdrücke teilte mir Frau Rosine Scheppler mit, in deren Besitz sich auch die jetzt nicht mehr gebrauchten Gerätschaften bebefinden: 1. on fait risi, rouir “ les fuade $d$ li (bottes de lin) sur un pré. 2. die kŭğs f. die Flachsbreche, die kleiner ist als die Hanfbreche, sert à kuēsę̧, à broyer le lin, kugsärr la femme qui le broie; ${ }^{1}$ die erix f. ,Acheln, chènevotte“ dienten als Brennmaterial. 3. der sre m. frz. séran sert à rifi le lin, c. à d. à le sérancer: der sre ist ein Brett, in dessen Mitte etwa ein Dutzend fingerlange eiserne Spitzen senkrecht im Kreise stehen: auf beiden Seiten ist eine Öffnung, durch welche Schnüre hindurchgezogen werden, um den sre festzubinden: der Flachs wird durch die Spitzen gezogen, gehechelt:

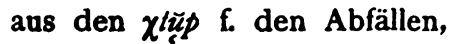
dem Werg, wird grobes Sacktuch hergestellt. 4. Der ş̣bră ist ein aufrecht stehendes, in einen Holzblock eingelassenes Brett, oben mit einwärts gebogenem Einschnitt: man legt die Flachsbündel über diese Krümmung und schlägt kräftig darauf mit der sufdžat f. (diese sieht einem grolsen Papiermesser ähnlich), um sie von Staub und kleinen sri $\mathrm{zu}$ säubern: dies heilst sųfdzi (von

1 In Strafsburg besitzt das Musée Alsacien verschiedene Exemplare von Flachs- und Hanfbrechen. 
suaviare?). 5. Gesponnen wird der Flachs an einem eigenen Spinnrad tr puc filg do $l i^{\sim}: k n a \bar{i}$ f. heilst der Spinnrocken, l\& trăt das Trittbrett, womit das $\mathrm{Rad}$ in Bewegung gesetzt wird, l\& spugl die Spule, lo fadi die hölzerne Einfassung der Spule mit eisernen Häkchen, über welche der Faden läuft; hēbl endlich ist der Flachshaspel.

lo Artikel und Pronomen mascul. singular., lo vănla $=$ le voilà. lẹ Ort; dže vii ale \& kẹ loe quelque part, \& pŭğ $d$ le nulle part; $s \check{a} i \sim$ bŭ lọ un bon terrain, guter Ackerboden.

luẹédz $\mathrm{m}$. le loyer, Miete.

lïn Mond ist die in Belmont übliche Form, nicht lin XXXIII, 4 I $u$. passim.

mabr Glied, nicht mab XXXIII, 412. $m \bar{a} m, m$ ợ $m$ même, nicht nur in Verbindung mit einem Pronomen lï $m$., sondern auch le mạm tsoz, lo mạm $q b i$.

mat kommt neben mate als Infinitiv = mettre vor; $z u \quad m a ̆ t$ gri s. gri.

me mich in me vănsi, me vănla. $m \varepsilon$ s. $t 5 q m$.

$m e ̨ r t s a \bar{a}(a / o)$ nicht mertša $\mathrm{XXX}$, 3 I 3 , petits escarbots noirs qui en courant font un mouvement de la tête. $m \bar{o} r$ mahlen neben mur. morăt f. petite truie. morī $\chi$ morille, s. küls.

mơii le mugissement faible, plaintif de la vache, dagegen $b r_{\bar{g}} r$, 3. $b r \bar{g}$ vom lauten Brüllen (zu XXXIII, 4I 3).

muzï m. das Mittel, le moyen. muzlā, f. muzlāer, sournois, ne disant rien, prenant un air

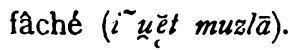

muzle être sournois, avoir l'air fâché.

$m u \bar{a}$; lo gro muā (le gros tas) sagte man früher für , hundert', allenfails noch $d u$ gro mūa für zweihundert (doch nicht weiter für 300); z. B. beim Verkauf einer Kuh hat jemand den, gro mila $\bar{a}^{\triangleleft}$ bekommen.

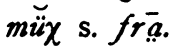

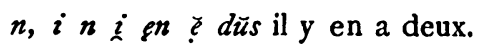
naxi noircir, schwärzen, z. B. $l i$ da $\bar{i}$ die Finger, den Ofen, usw.

nạmi nicht wahr? nạmm XXXIII, 414 ist in Belmont nicht üblich.

natej (le vęts se n.) 3. zu nătïi; s. natiōer.

natī̄er f. Nachgeburt, grandes peaux, grandes enveloppes que la vache expulse 5 à 8 heures après avoir vêlé; lokalfranzösisch: le chevet.

$n i$ Nest; 0 ke $n i$ von einem Zimmer, in dem alles in Unordnung ist.

$n i \bar{a}$ m. Nestei: i fa le leqi lo niāa. $n \bar{o} \mathrm{~m}$. auch Trog, auge du cochon, nō de pūō, Plur. di priè. 
ärsp m. Igel.

uẹd damner à une amende, s. \&mạn.

papingt f. Kochlöffel zum Umrühren (dasselbe wie grmonuat). pẹtlle m. le chiffonnier qui vient trois fois par an dans le village: on lui apporte les $p_{c}^{\breve{e} t}$ (vgl. XXXIII, 417 v. 3 pect) qu'on troque contre de la vaisselle et autres objets utiles au ménage.

pislę $d \check{z}$ pucelage.

piane m. petit pian (érable).

poii (se) pouiller.

poíu, f. poinuz pouilleux.

pota $d$ tsabr pot de chambre,

prta $d$ brkă pot de fleurs, potă $d$ lesse Milchtopf; s. küts. pür ,Birne' hörte ich zuletzt, nicht pür XXXUII, 41 8, s. $\& d \bar{a}-$ $d z i$; dagegen pürde $e^{i}$ s. v. pusi s. bra.

pupse $e^{i}$ auch tetins de chattes, de chiennes, etc.

purfiei $d$ li paquet de lin.

pü̈slā, f. püslēer qui cherche les puces.

püsle, I. püsẹl chercher les puces.

rại (besser als rāi XXXIII, 4I 9 )

instrument pour sortir la braise du four.

rạkinā, f. rạkināer qui cherche

à jouer des tours aux autres. raú Infin. ravoir, Indic. Prés.

6. ra, Part. revü. r. Böschung ist maskulin.

rejg Plur. (li r $\bar{g} g$, li răg) Verschlag aus Holzstämımen oben in der Scheune zur Unterbringung des $\chi \rho$ chaume pour le toit (wird wenig mehr gebraucht, da heutzutage die meisten Häuser mit Ziegeln oder Schiefer gedeckt sind). rexęcp, Part. rexępü, r. \& lq mūọ dem Tode entgehen; $t a$ r $r$ -

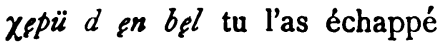
belle.

rexkrepii (se), 'hi \& vü s rexkrepizi (je les ai vus); bẹt rexkrẹ$p i e i$, se dit des poules qui se ramassent sur elles-mêmes, se serrent l'une contre l'autre sous la pluie.

rezong; \&n me rezon mi , ne me réplique pas'.

rguokf contrarier, agacer; ęn me revok mi.

rhăk (i) il s'assoupit ist XXXIII, $420 \mathrm{zu}$ streichen.

rid $z$ nennt man auch das Mehl. sieb.

rifi murs es XXXIV, 172 heilsen, nicht rif\&; s. auch rifi s. v. lic.

I. risi arracher en tirant avec force: risi red (fest), fice (heraus-), $b_{\xi}$ (herunter-), govq (weg-) reifsen.

2. risi s. $l i \sim$.

rǐğm, (nicht riģm XXXIII, 420), rięrmăt, auch petite bande d'un champ. 
rödž bœuf tout rouge XXXIII, 420 ; statt dessen braucht man meist fürỉ̇.

rọtg $i k$ d lāt éloigner l'un de l'autre.

rufgii (lg) regain, besser als rugai XXXIII, 421.

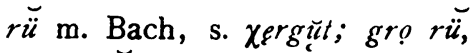
pti r $\breve{\ddot{u}}$ auch vom Wasser, das in der Gosse raiăl dahin schiefst.

riing (il ă), d'un champ mal soigné.

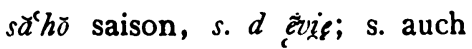
desa'hnāi.

serkig s. biklq.

siă gewils, me siă să vră mais certainemeni c'est vrai.

slïh f. Kirsche; slīh $d$ atr $\tilde{\xi}$ Moosbeere: die reife Frucht ohne Stein ist rund, rot und gleicht der Preifselbeere: sie hat aber viel kleinere Blätter und hängt an einem haardünnen Stielchen. Sie reift im Herbst auf den huble (s. dies) des Hochfeldes, überwintert unter dern Schnee und wird oft erst im Frühling gepflückt. Angeblich wird sie so genannt, weil ihr Genufs leicht berauscht (donne de l'entrain); einige halten sie für giftig.

slọ (neben slŏ), lo slọ ve bq die Sonne geht unter.

sọbră s. lĩ.

spvọ souvent. suar, man sagt bo suar, nicht să; s. žur.

sugdža

suedzi $\}$ s. lĩ.

sür (sa bi sürr) sicher.

$z u k \bar{a} i$ f. coup, poussée brusque contre quelque chose: $\check{z} m \varepsilon$

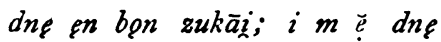
en bon zukāi evo sọ pụ̆ (son poing).

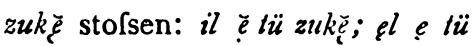
zukāị; il $i$ ẹ tü zukg epre nọt puōt.

šign Hündin sagt man, nicht tšięn XXXIII, 428 und 388 v. alg.

slurps vieille savate.

šŭk Ausruf, wenn man die Empfindung der Kälte hat, $i f_{\varepsilon}$ $\zeta \breve{u} k$; dagegen $s \not c k$ wenn man sich brennt, sqgk lo $n_{\xi}$, sp̧k if pèct.

spigl kleine Spule des mte (métier à tisser le ruban).

spugl s. lĩ.

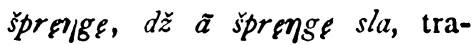
vailler rude et vite.

stọgr, auch šlaugr f. coup, bosse, ęn $\check{s} . \&$ lo tệt.

$t a l_{\xi}$, donner une ecchymose, $\check{z}$ $m$ \& tale.

talēer f. ecchymose, écorchure non ouverte; auch von Früchten, 
die $z \mathfrak{u}$ Boden fallen, und an einer Stelle eingedrückt und weich werden.

tar $\ddot{i}$ agacer, contrarier.

te toi, tu, Pronom. conjunctum und absolutum: te tsat, te vănla; vor Vokal $t, t a d \mathrm{du}$ bist.

terleps richtiger als tírlips XXXIII, 425.

tęts f. Stelle im Wald, wo man reichlich Beeren, Pflanzen findet: $d \ddot{z}$ a trove en bon tęts de brẹbel, $d$ epęm, de 'hnẹt (it. macchia); sonst heifst der Platz im Dorfe oder im Zimmer piess.

tn̄er: lo tn̄er e tše tsü lo bo: la foudre est tombée sur l'arbre. $t \bar{o} \dot{j}$ dü $\chi k$ $t \breve{a}$ sagt man zu einem Tauben (sourd que tu es): $t$ $\bar{\rho} \dot{i}$, du hörst' ist zum Hauptworte geworden.

toje $\mathrm{m}$. nourriture pour les cochons: légumes, feuilles de betteraves, carottes, petites pommes de terre - le tout écrasé et mêlé ensemble.

tokelg: XXXIII, 425, 1. Spalte, Z. I v. unten lies $k a p d e$ tokele statt $a t$.

trăt s. li.

trem s. xfuat.

trȩ̄r melken.

tribüstg inciter, pousser les gens au travail; te $m$ tribiust $q d e$.

trüts f. pied de salade, de seigle sur lequel plusieurs tiges sont réunies, ęn bol trüts. trütši Infin. u. Part. zu der XXXIV, 174 verzeichneten 3 . sing. trüts, von der Feldfrucht, die gedeiht, dicht steht (plusieurs tiges réunies sur un pied): lo sāl $n$ trütš mi, in $n$ ẹ mi trütši, $i$ trutšrẹ ko il deviendra encore touffu.

türbing se dit d'une femme qui trime dans le ménage. $t s ̌ d z i$ charger, hat auch die Bedeutung empierrer: ęn rut tša-

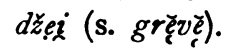

tsat $t_{\ell}$ zu dem veralteten Passé défini tsatce XXXIV, 174 hat nach Herrn Charles Morel der Plural tsătên, nicht tsatoen gelautet.

tsārlę $\mathrm{m}$. Bettstelle, bois de lit. tšăs f. pantalon, $t s \bar{a} s$ gu bęta (a/d) ancien pantalon avec pont, Hosenlatz.

tsāsăt f. Strumpf.

ts. $\overline{\tilde{c} m}$ f. Hanf. In Belmont wurde früher Hanf gebaut, heute nicht mehr: ein Teil der Gemarkung heifst noch $m_{\ell} d t s$ g m (hier heirst me nicht Garten). Auf die Hanfkultur und die Hanfbereitung bezügliche Ausdrücke sind nicht mehr erhalten. Die für den Flachs üblichen galten möglicherweise auch für den Hanf (z. B. kušcs, kucsse, s. li ), doch konnte ich darüber nichts bestimmtes erfahren.

tšermin (hāa $d_{d e)}$ buisson de charme. tšşsa m. porc moyen de 3 à 4 mois (Ferkel, das den Weg 
vom Markt bis zur Wohnung des Käufers zu Fufs zurücklegen kann).

$t_{\xi} \xi z a ̆ t$ א. petite cabane pauvre et délabrée (ungefähr was hibẹt);

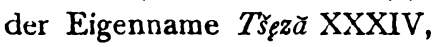
176 v. Chézd und ibid. S. 180 ist demnach casa + ittus, nicht $=$ afr. chasal.

tšñ $\check{L}_{\text {ăt }}$ f. aufser latrines auch Menschenkot: $d e$ ts. de vig chiasse de ver.

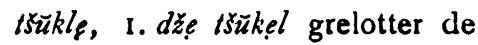
froid.

vănla s. vănsi.

vănsi und vănla sind neben văsi, vălă voici, voild gebräuchlich;

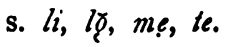

vartg espionner, guetter.

vǧxe m. Sarg.

vęrmin vermine.

A. Horning. 